\title{
Electrophysiological relationship between the nose and the hyoid bone complex
}

\author{
Susumu Mukai* \\ Mukai Clinic, Yamato, Kanagawa, Japan
}

\begin{abstract}
Objectives: The hyoid bone of the humans hangs in the upper part of the neck. It is connected to the styloid process via the stylohyoid ligament. The bottom edge of the hyoid bone the larynx is attached to the thyrohyoid ligament. In turn, the larynx is connected with the trachea, bronchi and lungs. These structures constitute a complex that is called the hyoid bone complex (HBC).Regulating the respiration HBC operates various human actions such as drinking, laughing, singing, swallowing, vomiting, crying and breathing. Upper movements of the HBC inhibit respiration whereas lower movements accelerate respiration.
\end{abstract}

Method: In this study, electrophysiological recordings were measured by using surface electrodes at the ala nasi and at the HBC during breath holding, phonation, breathing, swallowing and sniffing.

Results: Results showed that there was an electrophysiological relationship between the nose and the HBC during the examined functions.

Conclusion: Congruent movements and electrophysiological activity were observed in the two organs, suggesting that the both organs play an important role in essential human functions.

\section{Introduction}

The hyoid bone of the humans hangs in the upper part of the neck. It is connected to the styloid process via the stylohyoid ligament. The bottom edge of the hyoid bone the larynx is attached to the thyrohyoid ligament. In turn, the larynx is connected with the trachea, bronchi and lungs. These structures constitute a complex that is called the hyoid bone complex (HBC). As the hyoid bone is able to move upwards and downwards via the muscles that are attached to its top and bottom edge, the HBC is able to move upwards and downwards too [1]. Upper movements of the HBC inhibit respiration whereas lower movements accelerate respiration. Regulating the respiration HBC operates various human actions such as drinking, laughing, singing, swallowing, vomiting crying and breathing. In this study, to assess the relationship between the nose and the $\mathrm{HBC}$, electrophysiological recordings were studied during typical functions such as breathing, phonation, coughing, swallowing, and sniffing.

\section{Methods}

Electrophysiological recordings were performed in a consulting room. Surface electrodes were attached at the same level of both ala nasi (AN) and hyoid bone complex (HBC). Electrophysiological activity was recorded by using the "Neuropack S1" (MEB-9402MB, $2 / 4$ ch. EMG/EP Measuring System, Nihon Kohden Co.) (Figure 1). Six subjects were involved in the experiments ( 2 males, 4 females; age range: 26 to 48 years). Subjects were informed about the purpose of the study and about the protocol and the experiments were performed after they expressed their consent.

The following situations were studied:

1. Breath holding

2. Deep inspiration through the nose

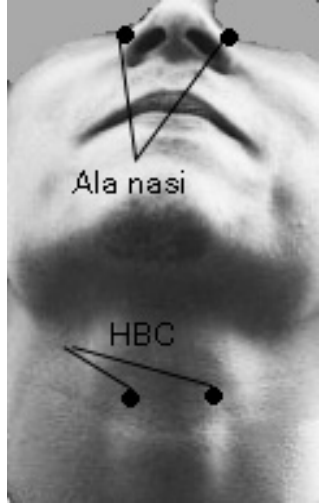

Figure 1. Electrode positions at the ala nasi (AN) and the hyoid bone complex (HBC)

3. Phonation $[\mathrm{A}:]$

4. Coughing

5. Swallowing

Swallowing was, in turn, divided into the following four stages:

- Beverage (coffee) held in the mouth

- Beverage swallowed

${ }^{\star}$ Correspondence to: Susumu Mukai, Mukai Clinic, Yamatominami 2-8-9, T242-0016, Yamato, Kanagawa, Japan; Email: s-mukai@da2.so-net.ne.jp

Key words: hyoid bone complex (HBC), ala nasi, EMG, breathing, swallowing, sniffing, coughing

Received: March 05, 2020; Accepted: March 18, 2020; Published: March 23 , 2020 
- Just after swallowing

- Coffee entered the esophagus

6.. Sniffing

\section{Results}

In this section, exemplary recordings from one of the study participants are shown in figures 2-7 (male subject, 35 years old). The same patterns were observed in all the participants

1. Breath holding: The Figure shows that low-voltage, fast waves were recorded from the AN (top waveform) and slower low-voltage, but slightly higher waves, were recorded from the HBC. The frequency of waves was $50 \mathrm{cycles} / \mathrm{sec}$ and may be related to power-line noise from electrical wirings in the room. (Frequency of electricity in Kanagawa district in Japan is $50 \mathrm{~Hz}$. The effect of noise is clearer on $\mathrm{HBC}$ recordings because the $\mathrm{HBC}$ electrode is more distant from the target structure than the AN electrode. There were no distinct discharge patterns except as only noise was measured from the AN and the HBC (Figure 2).

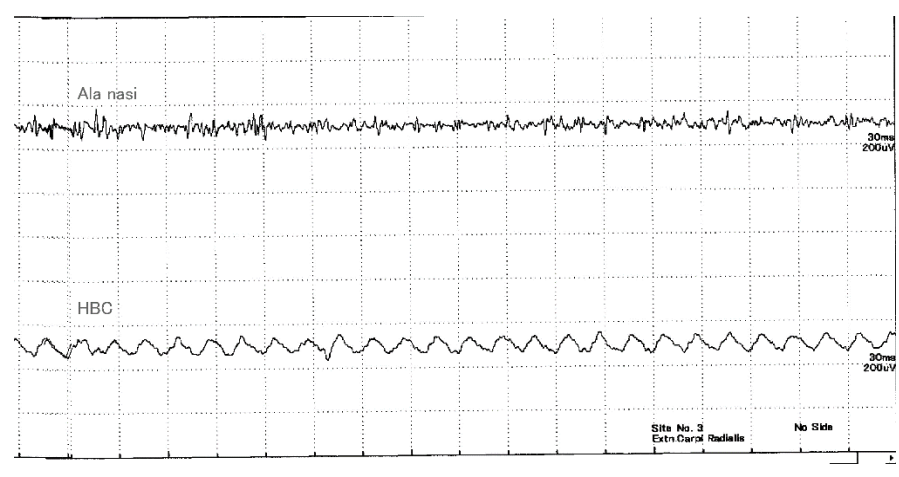

Figure 2. Recordings during breath holding

2. Deep inspiration through the nose: High voltage waves were observed from the AN when air was inhaled deeply through the nose. Relatively higher voltage than those of breath holding waves were observed from the HBC (Figure 3).

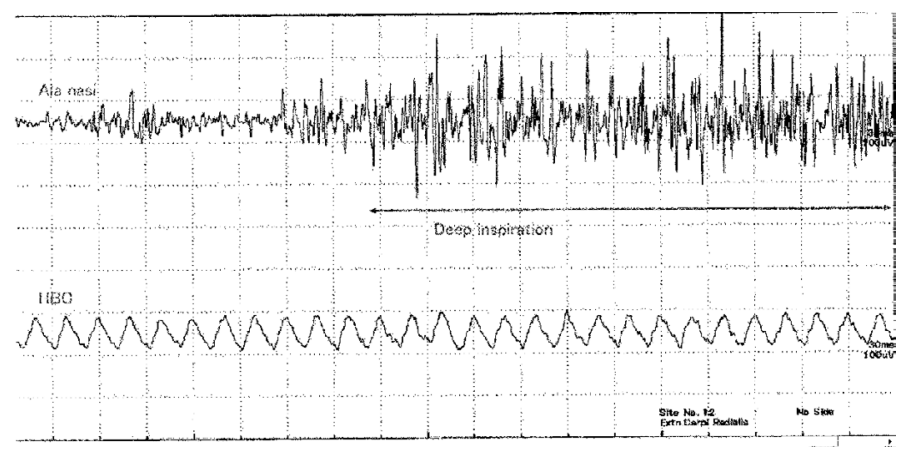

Figure 3. Recordings during deep inspiration from the nose

3. During phonation [A:]: Several high-voltage, high frequency spikes from muscles of the AN were observed during pronunciation of [A:]. Slightly higher waves were obtained from the HBC. Although laryngeal muscles are spiking during phonation, those were not recorded from the HBC (Figure 4).

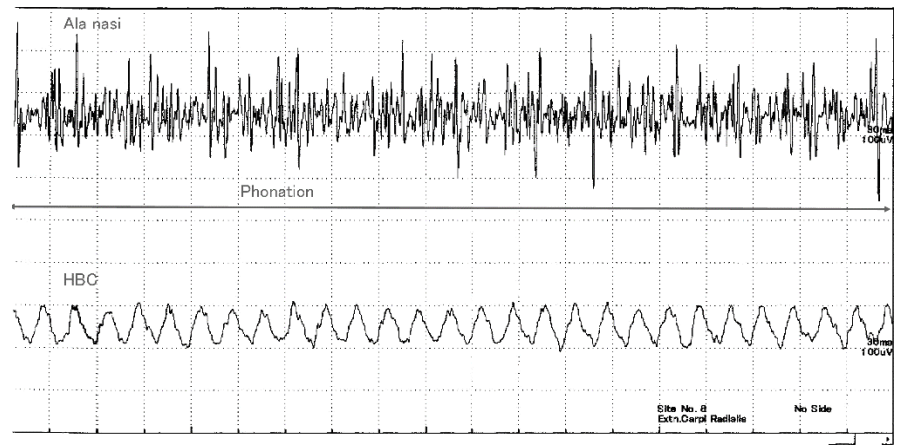

Figure 4. Recordings during phonation [A:]

4. When coughing: Waves with irregular voltage patterns were obtained from the AN at about $40 \sim 50 \mathrm{~ms}$ after coughing. From the $\mathrm{HBC}$, unstable waves were observed before coughing and higher waves appeared during coughing (Figure 5).

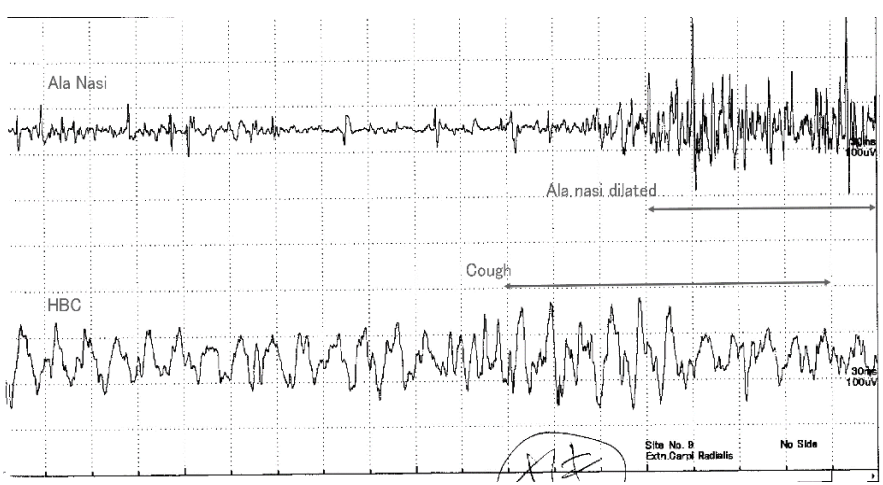

Figure 5. Recordings when coughing

5. Coffee was used in this swallowing study. Swallowing was divided into four stages as follows:

- Beverage (coffee) held in the mouth: When holding coffee in the mouth, low irregular spikes were observed at the AN. No apparent discharge was observed from the HBC (Figure 6A).

\section{$6 \mathrm{~A}$}

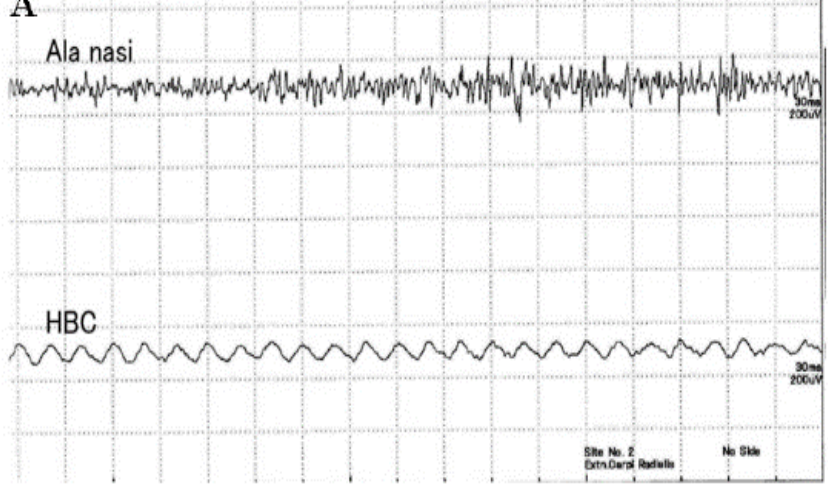

Figure 6. Recordings during swallowing

6 A) Beverage (coffee) held in the mouth

- Beverage (coffee) swallowed: During swallowing, no spikes were observed from the AN. High voltage, relatively regular spikes were observed from the HBC (Figure 6B). 


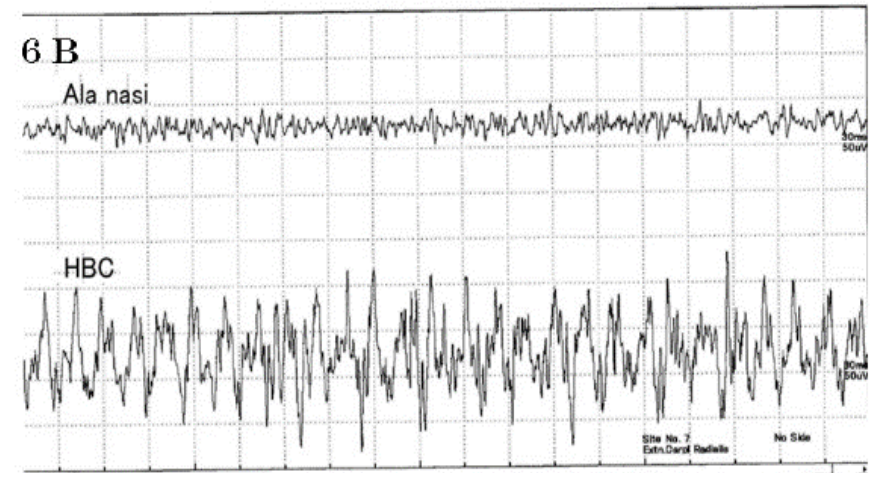

Figure 6. Recordings during swallowing

6 B) Beverage (coffee) swallowed

- Just after swallowing : no spikes were observed during swallowing from the AN. From about $105 \mathrm{~ms}$ before swallowing, the muscle activity of AN started and continued for about $330 \mathrm{~ms}$ after swallowing. It can be thought that breathing restarted at this time. Waves from the $\mathrm{HBC}$ returned to a resting state after swallowing (Figure 6C).

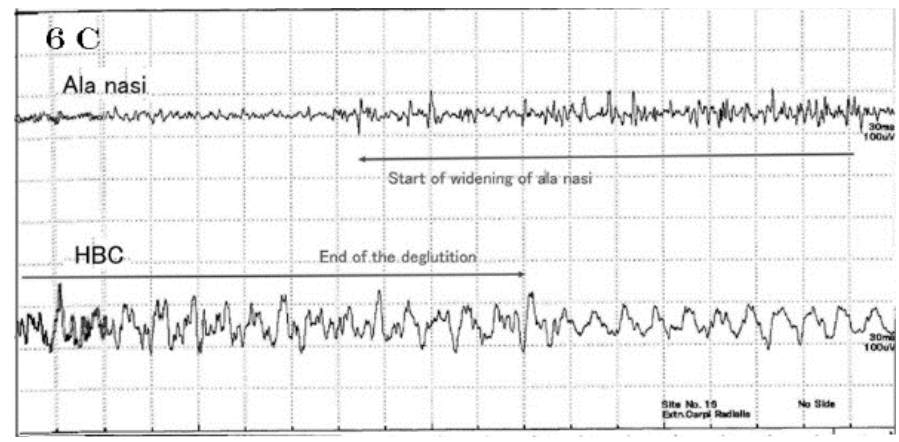

Figure 6. Recordings during swallowing

6 C) Just after swallowing

- Coffee entered into the esophagus : Both $\mathrm{AN}$ and $\mathrm{HBC}$ returned to their resting state (Figure 6D).

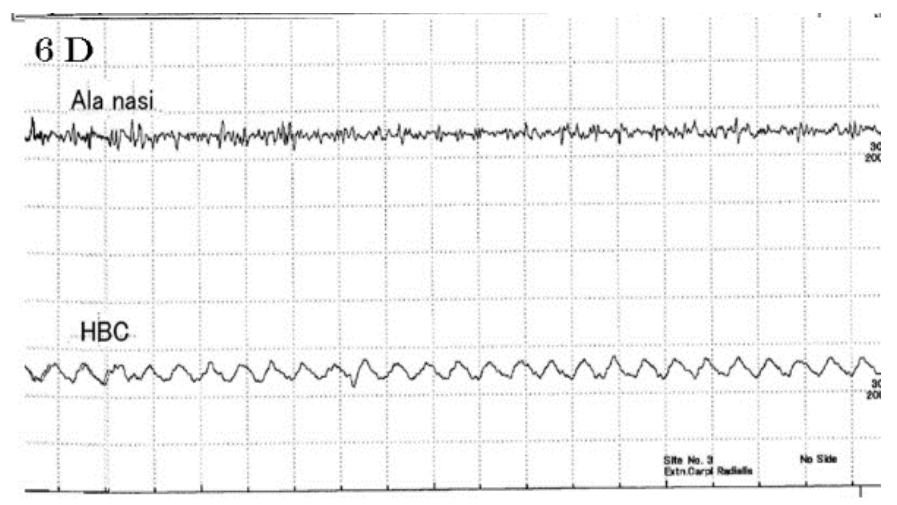

Figure 6. Recordings during swallowing

$6 \mathrm{D})$ Coffee entered the esophagus

6. Sniffing: From about 10 to $20 \mathrm{~ms}$ before sniffing, active potentials were observed from the AN. Then the $\mathrm{HBC}$ was activated for almost the same interval (Figure 7).

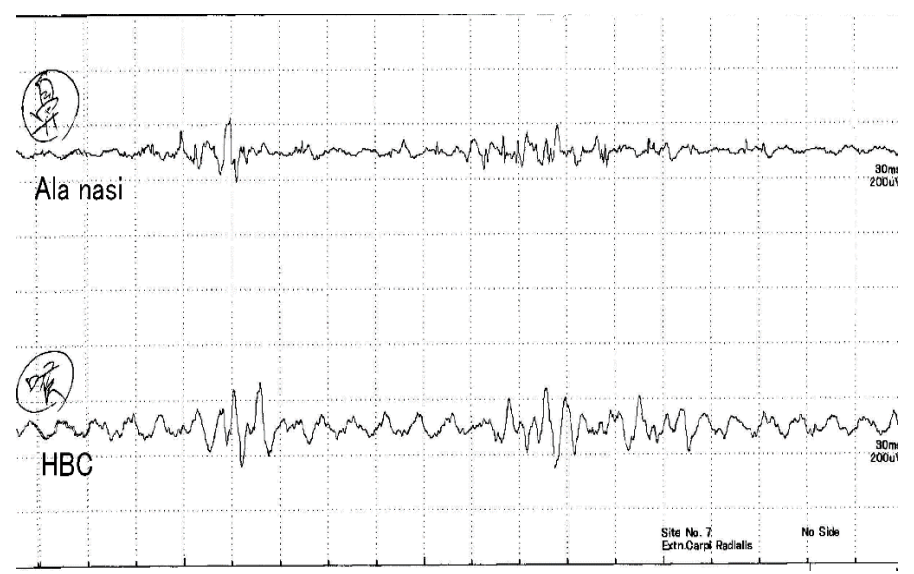

Figure 7. Recordings during sniffing

\section{Discussion}

When breath was held no apparent electrical discharge was observed from the AN and $\mathrm{HBC}$, suggesting that both the nose and the HBC were inactive. They are in a static open state while breath is held (Figure 2). High spikes were observed at the AN when air was deeply inhaled through the nose (Figure 3). This is in line with previous studies that demonstrated that when the glottis opens wide, the AN open wide, as the activities of posterior crico-arytenoid muscles are synchronous with AN muscles [2-4]. This study showed that discharges from the HBC increased during deep breaths, suggesting that the HBC moved downward to enable respiration. This suggests that the nose, the larynx and the HBC opened wide to inhale more air. The fact that the nose opens wider may suggest, in turn, that the body is trying to further decrease temperature in the brain $[5,6]$. Active discharges were observed from both AN and HBC during phonation of [A:] (Figure 4). Although the laryngeal muscles adduct during vocalization, discharges from them were not clear from the $\mathrm{HBC}$ recording. This suggests that discharges from the laryngeal muscles are not detected by the surface electrode at the HBC. The HBC moves downward during phonation, meaning that respiration is involved. In addition, active discharges from the nose are related to the fact that the respiratory tract of the $\mathrm{HBC}$ is wide open. These observations suggest that the structures of the nose and the $\mathrm{HBC}$ open wider for vocalization. They might act as voice resonators.

Highly irregular voltage spikes were observed from the AN from $40 \sim 50 \mathrm{~ms}$ after coughing (Figure 5). As opposed to the discharge observed from the AN, from the HBC unstable waves were observed before coughing and high waves during coughing. These observations suggested that the AN may not move before coughing. The nose opens wide from 40 to $50 \mathrm{~ms}$ after coughing, and the HBC moves before coughing and then it starts moving vigorously when coughing begins. From a physiologically perspective, coughing, defecation or effortful action such as weightlifting elicit protective movements of the larynx towards the lower airways and abdomen [7-9]. The larynx might be closed tight by upward moving of the HBC. These movements can be detected by the fingers on the neck too.

Downward movements of the HBC accelerate respiration [1] Opening of the AN means that the glottis opens wider, too. These movements suggest that both the airway and nose work to increase the airflow and that airways are cleansed when coughing. 


\section{Electrophysiological observations during swallowing were divided into four stages (Figure 6; 1 4):}

- When coffee was held in the mouth, low irregular spikes were observed at the AN. No spike was observed from the HBC. When coffee was held in the mouth, nasal breathing continued whereas the $\mathrm{HBC}$ remained in its resting state (Figure 6A).

- When swallowing (Figure 6B), no discharge was observed from the AN. On the contrary, high and active discharges were observed from the HBC. Neither inhalation nor exhalation of air is possible during swallowing. The nares stop their movements. Only the $\mathrm{HBC}$ is active during swallowing. The HBC moves upwards, and it shrinks against the entry of any foreign body into lower airways by the laryngeal reflex closure [10]. In fact, organisms cannot inhale nor exhale air during this phase.

- No action potential was observed from the AN when swallowing (Figure 6C). Activities of the nostrils restarted about $105 \mathrm{~ms}$ before the end of swallowing. They continued during about $330 \mathrm{~ms}$ after swallowing as observed from the AN. It was thought that rebreathing started at this time. Waves from the $\mathrm{HBC}$ returned to the resting state after swallowing. Both the AN and the $\mathrm{HBC}$ returned to resting state after beverages entered into the esophagus (Figure 6D) [11-14].

- As observed by fiberscope, vocal folds open and narrow with sniffing rhythms [15]. In this study, from 10 to $20 \mathrm{~ms}$ before sniffing active potentials were observed from the AN. Then the HBC became activated at almost the same duration. Active potentials from the HBC were not from the vocal fold as seen in phonation.

The observed discharges from both the AN and HBC meant that after the nose opened wider for sniffing, the HBC moved with the same sniffing rhythms. The movement of the HBC can be thought to be downwards as these movements accelerate respiration. That is, airways from the nose to the trachea open wider for sniffing. As such, these movements might contribute to the sense of smell [16].

\section{Conclusion}

Electrophysiological recordings from surface electrodes at the level of AN and HBC showed that both the AN and HBC are static when respiration stops, whereas they open wide during breathing and phonation. Recordings showed that the AN stops its activities and the $\mathrm{HBC}$ is active during swallowing, whereas both organs open wide after swallowing. Moreover, results showed that the AN and $\mathrm{HBC}$ are active when sniffing. Overall, this study suggested that, physiologically the $\mathrm{AN}$ and the $\mathrm{HBC}$ are related and play a key role in functions that are essential to life.

\section{References}

1. Mukai S (2018) The hyoid bone complex. Zetuyuchaksho Kenkyuukai Kaiho:12-21.

2. Mann DG, Sasaki CT, Suzuki M, H. F, Hernandez JR (1977) Dilator naris muscle. Ann Otol Rhinol Laryngol 86: 362-370. [Crossref]

3. Suzuki M, Kirchner JA (1969) Posterior cricoarytenoid as an inspiratory muscle. Ann Otol 78: 154-158.

4. Suzuki M, Kirchner JA, Murakami Y (1970) The cricothyroid as a respiratory muscle Its characteristics in bilateral recurrent laryngeal nerve paralysis. Ann Otol Rhinol Laryngol 79: 976-983. [Crossref]

5. Mukai S (2015) Human nose might be involved in cooling of the brain. Int J Clin Med 6: $482-486$

6. Mukai S (2017) Amelioration of sleep by expansion of the nasal cavity: A role of the nose. Ann Clin Otolaryngol 2: 1015.

7. Negus V (1929) The mechanism of the larynx. London: WM Heinemann Ltd.

8. Fink BR (1975). The human larynx; a functional study. New York: Raven Press.

9. Suzuki M, Sasaki T (1976) Initiation of reflex glottic closure. Ann Otol Rhinol Laryngol 85: 382-386. [Crossref]

10. Mukai S (1976) Functional relationship between post-crycoarytenoid muscles and diaphragm. J Broncho Esophago Soc 27: 300-304.

11. Shin T (1994) Relationship between swallowing and respiration. Ji-Bi 40: 332-363.

12. Shin T, Umezaki T (1995) Swallowing function test based on neural mechanisms. Practica Oto-Rhino-Laryngologica 88: 553-559.

13. Kim SM, McCulloch TM, Rim K (2000) Pharyngeal pressure analysis by the finite element method during liquid bolus swallow. Ann Otol Rhinol Laryngol 109: 585-589. [Crossref]

14. Amirali A, Tsai G, Weisz D, Schrader N, Sanders I (2001) Mapping of brain stem neural circuitry active during swallowing. Ann Otol Rhinol Laryngol 110: 502-513. [Crossref]

15. Mukai S (1989) Vocal cord adduction while sniffing. Arch Otolaryngol Head Neck Surg 115: 1362-1366. [Crossref]

16. Mukai S (1989). Four cases of dysosmia with no vocal cords adduction during sniff (laryngeal dysosmia). Nihon Jibiinkoka Gakkai kaiho 92: 1211-1219. [Crossref]

Copyright: (C2020 Mukai S. This is an open-access article distributed under the terms of the Creative Commons Attribution License, which permits unrestricted use, distribution, and reproduction in any medium, provided the original author and source are credited. 